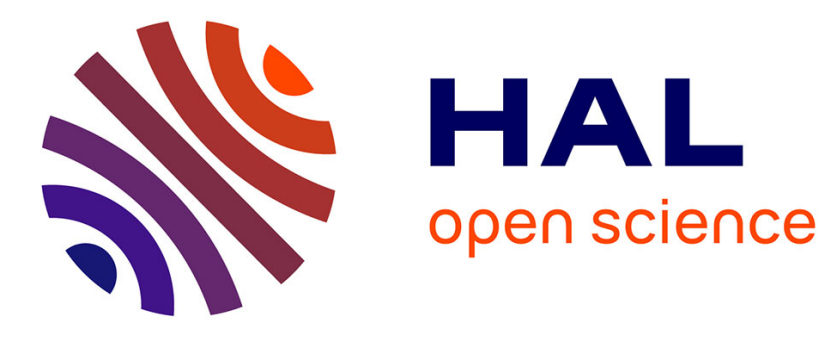

\title{
Robust Multiuser Binary CPM Detection with Unkown modulation index
}

Malek Messai, Frédéric Guilloud, Karine Amis Cavalec, Giulio Colavolpe

\section{To cite this version:}

Malek Messai, Frédéric Guilloud, Karine Amis Cavalec, Giulio Colavolpe. Robust Multiuser Binary CPM Detection with Unkown modulation index. EUSIPCO 2015 : 23nd European Signal Processing Conference, Aug 2015, Nice, France. pp.2132-2136, 10.1109/EUSIPCO.2015.7362761 . hal-01288308

\section{HAL Id: hal-01288308 https://hal.science/hal-01288308}

Submitted on 14 Mar 2016

HAL is a multi-disciplinary open access archive for the deposit and dissemination of scientific research documents, whether they are published or not. The documents may come from teaching and research institutions in France or abroad, or from public or private research centers.
L'archive ouverte pluridisciplinaire HAL, est destinée au dépôt et à la diffusion de documents scientifiques de niveau recherche, publiés ou non, émanant des établissements d'enseignement et de recherche français ou étrangers, des laboratoires publics ou privés. 


\section{Robust Multiuser Binary CPM Detection with Unkown modulation index}

\author{
Malek MESSAI, Frédéric GUILLOUD, Karine AMIS \\ Signal and Communication Department of Telecom Bretagne-Institut \\ Telecom, CNRS Lab-STICC (UMR 6285), Brest 29200, France \\ e-mail: firstname.lastname@telecom-bretagne.eu
}

\author{
Giulio COLAVOLPE \\ Università di Parma, Dipartimento di \\ Ingegneria dell'Informazione, Viale G. P. Usberti, \\ 181A, I-43124 Parma, Italy, e-mail: giulio@unipr.it.
}

\begin{abstract}
We develop a robust multiuser detector for a Frequency Division Multiplexing (FDM) system where each user employs a binary continuous phase modulation (CPM) generated through a low-cost transmitter, thus characterized by a significant modulation index uncertainty, and sent over a channel affected by phase noise. In this FDM system the spectral efficiency can be increased by reducing the spacing between two adjacent channels. The proposed receiver is designed by adopting a simplified representation of a binary CPM signal with the principal component of its Laurent decomposition and is obtained by using the framework based on factor graphs and the sum-product algorithm. This detector can be used for iterative detection/decoding of a coded scheme in which each user employs a binary CPM modulator serially concatenated with an outer encoder through a pseudo-random interleaver. It does not require an explicit estimation of the modulation index nor of the channel phase and is very robust to large uncertainties of the nominal value of the modulation index.
\end{abstract}

Index Terms-Continuous phase modulation, CPM-FDM, frequency spacing, spectral efficiency, modulation index mismatch, phase noise, factor graph, sum product, iterative detection and decoding.

\section{INTRODUCTION}

In frequency division multiplexed (FDM) systems, the spectral efficiency (SE) can be increased by reducing the spacing between adjacent users, thus allowing overlap in frequency and hence admitting a certain amount of interference. This aspect has been investigated from an information-theoretic point of view for continuous phase modulations (CPMs) [1], showing that a significant improvement can be obtained even for a single-user detector. The benefits in terms of spectral efficiency can be even larger when a multiuser receiver is adopted. Optimal multiuser detectors have a complexity which increases exponentially with the number of users, therefore suboptimal detection schemes are required. Recently a reducedcomplexity MUD algorithm for an additive white Gaussian noise (AWGN) channel has been derived in [2] based on factor graphs (FGs) and the sum-product algorithm (SPA) [3]. The algorithm designed in [2] based on this framework outperforms all other suboptimal MUD algorithms both from performance and complexity points of view.

We here focus on FDM systems where each user employs a binary CPM (FDM-CPM). CPMs are well known by their constant envelope that makes these modulations insensitive to nonlinear distortions and thus very attractive for applications in satellite communications and in low-cost and low-power consumption transmitter standards. An analog implementation of the CPM modulator allows to further reduce the transmitter cost, at the expense of possible variations of the CPM waveform parameters around their nominal values. In particular, the modulation index will vary since it depends on the not well calibrated gain of the employed voltage-controlled oscillator (VCO). As an example, in Bluetooth operating in Basic Rate (BR) and Low Energy (LE) modes, the modulation index is specified to be in the intervals $[0.28,0.35]$ and $[0.45,0.55]$, respectively [4]. The interval of the modulation index for the Digital Enhanced Cordless Telecommunication Ultra Low Energy (DECT ULE) is $[0.35,0.7]$ [5]. In the Automatic Identification System (AIS), the modulation index is nominally equal to 0.5 but due to the imperfections of the AIS equipments, a variation of $\pm 10 \%$ is typically admitted [6]. In this paper, we consider the very general problem of multiuser detection of binary CPM signals with an unknown modulation index transmitted over a channel with phase noise. We adopt a simplified representation of the CPM signal based on the principal component of its Laurent decomposition [7] and describe the joint a-posteriori probability of the transmitted symbols, the channel phase, and the modulation index through a factor graph (FG) [3]. The sum-product algorithm (SPA) is then advocated to compute the a-posteriori probabilities of the transmitted symbols. In particular, we will consider binary CPMs serially concatenated with an outer code through an interleaver, and iterative detection/decoding.

This paper is organized as follows: After the system model description in Section II, we rederive the FG-Multiuser detector proposed in [2] by using Laurent's decomposition in Section III. This algorithm is extended to the case where each user's signal is generated with an unknown modulation index mismatch and transmitted over a phase noise channel in Section IV. Simulations results are discussed in Section V and finally some conclusions are drawn in Section VI.

The new multiuser receiver for binary CPM signals which is robust to the modulation index mismatch even in the presence of phase noise is named "robust FG-MUD". 


\section{System Model}

The signal model is a synchronous $K$-user CPM system in which all users share the same modulation format and transmit at the same power. The extension to the case of asynchronous users, possibly with different power and modulation format can be pursuaed as described in [2]. We assume that each user transmits $N$ information bits and we denote by $a_{n}^{(k)}$ the $n$-th symbol from the $k$-th user, whose value is drawn from the set $\{-1,1\}$. The sequence of symbols transmitted by the $k$-th user is $\boldsymbol{a}^{(k)}=\left(a_{0}^{(k)}, a_{1}^{(k)}, \ldots, a_{N-1}^{(k)}\right)$. We define $\boldsymbol{a}_{n}=\left(a_{n}^{(1)}, a_{n}^{(2)}, \ldots, a_{n}^{(K)}\right)$ and we denote by $\boldsymbol{a}=\left(\boldsymbol{a}_{0}^{T}, \boldsymbol{a}_{1}^{T}, \ldots \boldsymbol{a}_{N-1}^{T}\right)^{T}$ the $K \times N$ matrix of transmitted symbols ${ }^{1}$. The complex envelope of the received signal can be written as

$$
r(t)=\sum_{k=1}^{K} s^{(k)}\left(t, \boldsymbol{a}^{(k)}\right) \exp \left(j 2 \pi f^{(k)} t\right)+\omega(t)
$$

where $\omega(t)$ is a complex zero-mean circularly symmetric white Gaussian noise with power spectral density $2 N_{0}, f^{(k)}$ is the difference between the carrier frequency of user $k$ and the reference frequency. $s^{(k)}\left(t, \boldsymbol{a}^{(k)}\right)$ is the binary CPM information-bearing signal of user $k$ which is given by

$$
s\left(t, \boldsymbol{a}^{(k)}\right)=\sqrt{\frac{2 E_{b}}{T}} \exp \left\{j 2 \pi h \sum_{n=0}^{N-1} a_{n}^{(k)} q(t-n T)\right\},
$$

where $E_{b}$ is the energy per information bit, $T$ the bit interval, $h$ the modulation index, the function $q(t)$ is the phase-smoothing response, and its derivative is the frequency pulse, assumed of duration $L T$. Based on Laurent representation, the complex envelope of the CPM signal may be exactly expressed as [7]

$$
s\left(t, \boldsymbol{a}^{(k)}\right)=\sum_{i=0}^{2^{(L-1)}} \sum_{n} \alpha_{i, n}^{(k)} p_{i}(t-n T)
$$

where the expressions of pulses $\left\{p_{i}(t)\right\}$ and those of symbols $\left\{\alpha_{i, n}^{(k)}\right\}$ as a function of the information symbol sequence $\left\{a_{n}^{(k)}\right\}$ may be found in [7]. By truncating the summation in (3) considering only the first $Q<2^{(L-1)}$ terms, we obtain an approximation of $s\left(t, \boldsymbol{a}^{(k)}\right)$. Most of the signal power is concentrated in the first component, i.e., that associated with the pulse $\left\{p_{0}(t)\right\}$, which is called principal component [7]. As a consequence, the principal component may be used in (3) to attain a very good trade-off between approximation quality and number of signal components [8], [9]. In this case, it holds

$$
\alpha_{0, n}^{(k)}=\alpha_{0, n-1}^{(k)} e^{j \pi h a_{n}^{(k)}}
$$

When the modulation index is rational, i.e., it can be expressed as $h=r / p$, where $r$ and $p$ are relatively prime integers, symbols $\left\{\alpha_{0, n}^{(k)}\right\}$ take on $p$ values [7].

\footnotetext{
${ }^{1}$ In the following, $(.)^{T}$ denote transpose and $(.)^{H}$ transpose conjugate.
}

\section{FG-Multiuser Detection Based on LAURENT DECOMPOSITION}

In this section we adopt the algorithm proposed in [2] to multi-user FDM-CPM systems by using the Laurent representation of a CPM signal. Although the bandwidth of the CPM signal in eq. (2) is infinite, strictly speaking, we assume hereafter that it can be limited to $N_{s} / 2 T, N_{s}$ being a positive integer chosen such that the approximation is fair. Thus, a simpling rate yielding $N_{s}$ samples per symbols produces an approximated set of sufficient statistics [10] provided that the received signal has been pre-filtered through an analog low-pass filter having a vestigal symmetry around $N_{s} / 2 T$. Under these assumptions, the noise samples are i.i.d complex Gaussian random variables with with mean zero and variance $\xi^{2}=\frac{N_{0} N_{s}}{T}$. We denote by $r$ the vector of all received samples defined by $\boldsymbol{r}=\left(\boldsymbol{r}_{0}^{T}, \boldsymbol{r}_{1}^{T}, \ldots, \boldsymbol{r}_{N-1}^{T}\right)^{T}$ with $\boldsymbol{r}_{n}^{T}=\left(r_{n, 0}, r_{n, 1}, \ldots, r_{n, N_{s}-1}\right)$ and $r_{n, m}$ denotes the $m$-th received samples $\left(m=0,1, \ldots, N_{s}-1\right)$ of the $n$-th interval. Based on the application of the FG/SPA framework, a suitable factorization of the probability mass function $P\left(\boldsymbol{a}, \boldsymbol{\alpha}_{0} \mid \boldsymbol{r}\right)$ can be derived as follows

$$
P\left(\boldsymbol{a}, \boldsymbol{\alpha}_{0} \mid \boldsymbol{r}\right) \propto p\left(\boldsymbol{r} \mid \boldsymbol{a}, \boldsymbol{\alpha}_{0}\right) P\left(\boldsymbol{\alpha}_{0} \mid \boldsymbol{a}\right) P(\boldsymbol{a})
$$

where $\boldsymbol{\alpha}_{0}=\left(\boldsymbol{\alpha}_{0}^{(1)}, \boldsymbol{\alpha}_{0}^{(2)}, \ldots, \boldsymbol{\alpha}_{0}^{(K)}\right)$ with $\boldsymbol{\alpha}_{0}^{(k)}=$ $\left(\alpha_{0,0}^{(k)}, \alpha_{0,1}^{(k)}, \ldots, \alpha_{0, N-1}^{(k)}\right)$. Each term can be further factored as follows:

$$
\begin{aligned}
P(\boldsymbol{a}) & =\prod_{k=1}^{K} \prod_{n=0}^{N-1} P\left(a_{n}^{(k)}\right) \\
P\left(\boldsymbol{\alpha}_{0} \mid \boldsymbol{a}\right) & =\prod_{k=1}^{K} P\left(\alpha_{0,0}^{(k)}\right) \prod_{n=0}^{N-1} P\left(\alpha_{0, n+1}^{(k)} \mid a_{n}^{(k)}, \alpha_{0, n}^{(k)}\right) \\
p\left(\boldsymbol{r} \mid \boldsymbol{a}, \boldsymbol{\alpha}_{0}\right) & \propto \prod_{n=0}^{N-1} F_{n}\left(\boldsymbol{a}_{n}, \boldsymbol{\alpha}_{0, n}\right) \prod_{k=1}^{K} H_{n}^{k}\left(a_{n}^{(k)}, \alpha_{0, n}^{(k)}\right)
\end{aligned}
$$

where

$$
\begin{aligned}
P\left(\alpha_{0, n+1}^{(k)} \mid a_{n}^{(k)}, \alpha_{0, n}^{(k)}\right) & \propto I_{n}^{(k)}\left(\alpha_{0, n+1}^{(k)}, \alpha_{0, n}^{(k)}, a_{n}^{(k)}\right) \\
F_{n}\left(\boldsymbol{a}_{n}, \boldsymbol{\alpha}_{0, n}\right) & =\prod_{i=1}^{K-1} \prod_{j=i+1}^{K} \exp \left\{-\frac{1}{\xi^{2}} \Re\left[\boldsymbol{s}_{n}^{(i) H} \boldsymbol{s}_{n}^{(j)}\right]\right\} \\
H_{n}^{k}\left(a_{n}^{(k)}, \alpha_{0, n}^{(k)}\right) & =\exp \left\{\frac{1}{\xi^{2}} \Re\left[\boldsymbol{r}_{n}^{H} \boldsymbol{s}_{n}^{(k)}\right]\right\}
\end{aligned}
$$

with $I_{n}^{(l)}\left(\alpha_{0, n+1}^{(l)}, \alpha_{0, n}^{(l)}, a_{n}^{(l)}\right)$ the indicator function equal to one if $a_{n}^{(l)}, \alpha_{0, n}^{(l)}$ and $\alpha_{0, n+1}^{(l)}$ satisfy the trellis constraint for user $l$, and equal to zero otherwise. Hence, we finally have

$$
\begin{aligned}
& P\left(\boldsymbol{a}, \boldsymbol{\alpha}_{0} \mid \boldsymbol{r}\right) \propto\left[\prod_{k=1}^{K} P\left(\alpha_{0,0}^{k}\right)\right] \prod_{n=0}^{N-1} F_{n}\left(\boldsymbol{a}_{n}, \boldsymbol{\alpha}_{0, n}\right) \\
& \prod_{k=1}^{K} H_{n}^{k}\left(a_{n}^{(k)}, \alpha_{0, n}^{(k)}\right) I_{n}^{(k)}\left(\alpha_{0, n+1}^{(k)}, \alpha_{0, n}^{(k)}, a_{n}^{(k)}\right) P\left(a_{n}^{(k)}\right)
\end{aligned}
$$

The resulting FG, not shown here due to a lack of space, has cycles of length four. The application of the SPA to 
a FG with cycles allows an approximate (due to the presence of cycles) computation of the a posteriori probabilities $P\left(a_{n}^{(k)} \mid \boldsymbol{r}\right)$ required for the implementation of the MAP symbol detection strategy [11]. However, the presence of short cycles of length four makes the convergence of the SPA to good approximations of the a posteriori probabilities $P\left(a_{n}^{(k)} \mid \boldsymbol{r}\right)$ very unlikely [11]. It is possible to remove these short cycles by stretching $\alpha_{0, n}^{(k)}$ in $\left(a_{n}^{(k)}, \alpha_{0, n}^{(k)}\right)$. In other words, instead of representing variables $a_{n}^{(k)}$ alone, we define a new variable given by the couple $\left(a_{n}^{(k)}, \alpha_{0, n}^{(k)}\right)$. This transformation does not involve approximations, since the resulting graph preserves all the information of the original graph. The resulting FG has cycles of length twelve. Since cycles are still present, the SPA applied to this graph is iterative and still leads to an approximate computation of the a posteriori probabilities $P\left(a_{n}^{(k)} \mid \boldsymbol{r}\right)$ [11]. However, the absence of short cycles allows us to obtain very good approximations. As shown in [2], the resulting algorithm outperforms all other suboptimal MUD algorithms both from performance and complexity points of view.

\section{Presence of Modulation Index Mismatch AND PHASE NOISE}

We now consider a multiuser transmission of binary CPM signals where the signal of user $k$ is generated through a lowcost transmitter, thus characterized by a significant modulation index uncertainty denoted by $h_{e}^{(k)}$ and sent over a typical satellite channel affected by phase noise $\left(h_{e}^{\left(k^{\prime}\right)}\right.$ and $h_{e}^{(k)}$ are assumed independent for $k^{\prime} \neq k$ ). Here, the framework of FG and SPA will be used in a double way. In addition to its use for the derivation of an efficient multi-user receiver with reduced complexity [2], it will also be used to allow detection in the presence of unkown modulation index ${ }^{2}$ and of a time varying phase noise as proposed for a single user transmission in [12], to derive finally a robust multi-user detector named "robust FG-MUD". In this case, the complex envelope of the received signal can be expressed as

$$
r(t)=\sum_{k=1}^{K} e^{j \theta^{(k)}(t)} s^{(k)}\left(t, \boldsymbol{a}^{(k)}, h+h_{e}^{(k)}\right) e^{\left(j 2 \pi f^{(k)} t\right)}+\omega(t)
$$

where $\theta^{(k)}(t)$ is the phase noise affecting user $k\left(\theta^{(k)}(t)\right.$ and $\theta^{\left(k^{\prime}\right)}(t)$ are assumed independent for $\left.k \neq k^{\prime}\right)$ introduced by the channel, modeled as a continuous-time Wiener process with incremental variance over a signaling interval equal to $\sigma_{\Delta}^{2}$. Approximating the signal through its principal component only and exploiting the feature that the pulse of the principal component weakly depends on the modulation index [13], we

\footnotetext{
${ }^{2}$ The modulation index error generated by the $k$-th low cost transmitter $h_{e}^{(k)}$ is assumed unkown and modeled as a random variable with known distribution.
}

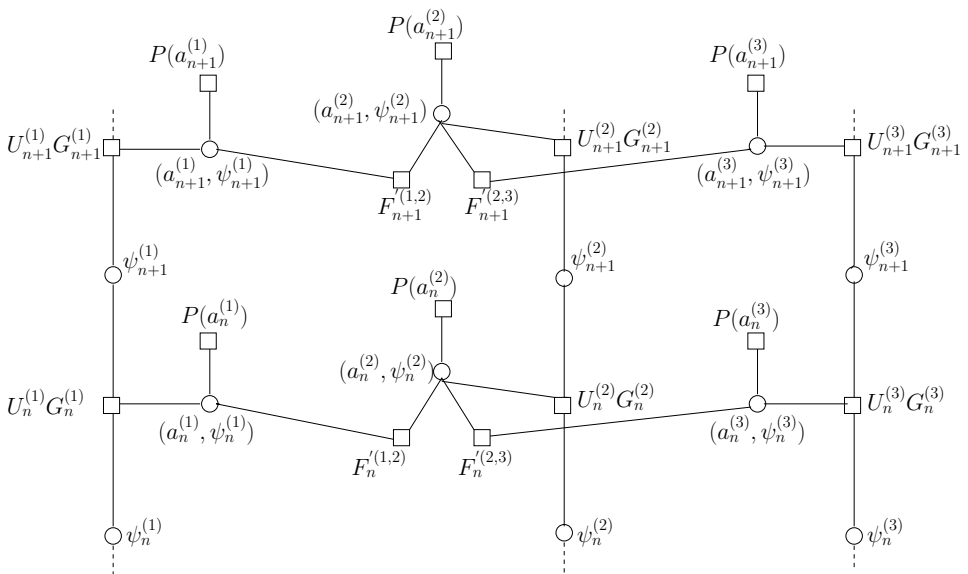

Fig. 1. FG corresponding to (14) and resulting from the approximation (17) for $K=3$ users.

can express the received signal as

$$
\begin{aligned}
r(t) & \simeq \sum_{k=1}^{K} e^{j \theta^{(k)}(t)} e^{j 2 \pi f^{(k)} t} \\
& \left(\sum_{n} \alpha_{0, n}^{(k)}(h) \alpha_{0, n}^{(k)}\left(h_{e}^{(k)}\right) \hat{p}_{0}(t-n T)\right)+\omega(t)
\end{aligned}
$$

where we have denoted by $\hat{p}_{0}(t)$ the pulse of the principal component for the nominal value of the modulation index $h$ and we have exploited the property, easily derived from (4), that symbol $\alpha_{0, n}^{(k)}$ related to the transmitted signal can be expressed as the product of symbol $\alpha_{0, n}^{(k)}(h)$ corresponding to the nominal CPM signal and symbol $\alpha_{0, n}^{(k)}\left(h_{e}^{(k)}\right)$ corresponding to a CPM signal with modulation index $h_{e}^{(k)}$. Under the assumption that the channel phase $\theta^{(k)}(t)$ is slowly varying such that it can be considered constant over the duration of pulse $\hat{p}_{0}(t)$, an approximate sufficient statistic may be obtained through a filter matched to pulse $\hat{p}_{0}(t)$. We define

$$
x_{n}^{(k)}=\int_{-\infty}^{+\infty} r(t) \hat{p}_{0}(t-n T) e^{-j 2 \pi f^{(k)} t} d t
$$

as the output, sampled at time $n T$, of the filter matched to $\hat{p}_{0}(t)$. We define also $\theta_{n}^{(k)}=\theta^{(k)}(n T), \phi_{n}^{(k)}=$ $\arg \left[\alpha_{0, n}^{(k)}(h)\right], \delta_{n}^{(k)}=\arg \left[\alpha_{0, n}^{(k)}\left(h_{e}^{(k)}\right)\right]$, and $\psi_{n}^{(k)}=\theta_{n}^{(k)}+$ $\phi_{n}^{(k)}+\delta_{n}^{(k)}$, collecting the samples of $\psi_{n}^{(k)}$ into a vector $\boldsymbol{\psi}_{n}=\left(\boldsymbol{\psi}_{n}^{(1)}, \psi_{n}^{(2)}, \ldots, \psi_{n}^{(K)}\right)^{T}, \boldsymbol{\psi}=\left(\boldsymbol{\psi}_{0}^{T}, \boldsymbol{\psi}_{1}^{T}, \ldots, \boldsymbol{\psi}_{N-1}^{T}\right)$, representing the received signal onto an orthonormal basis and denoting by $\boldsymbol{r}$ its vector representation. As the samples $\theta_{n}^{(k)}$ satisfy the discrete-time Wiener model

$$
\theta_{n}^{(k)}=\theta_{n-1}^{(k)}+\Delta_{n}^{(k)}
$$

where $\left\{\Delta_{n}^{(k)}\right\}$ are real, independent, and identically distributed Gaussian random variables, with zero mean and variance $\sigma_{\Delta}^{2}$, 
and $\theta_{0}^{(k)}$ is assumed uniformly distributed in $[0,2 \pi)$, it is thus

$$
\begin{aligned}
\psi_{n}^{(k)} & =\theta_{n}^{(k)}+\phi_{n}^{(k)}+\delta_{n}^{(k)} \\
& =\theta_{n-1}^{(k)}+\Delta_{n}^{(k)}+\phi_{n-1}^{(k)}+\pi h a_{n}^{(k)}+\delta_{n-1}^{(k)}+\pi h_{e}^{(k)} a_{n}^{(k)} \\
& =\psi_{n-1}^{(k)}+\Delta_{n}^{(k)}+\pi h a_{n}^{(k)}+\pi h_{e}^{(k)} a_{n}^{(k)} .
\end{aligned}
$$

Discarding the terms independent of symbols and states and taking into account that a CPM signal has a constant envelope, the joint distribution $p(\boldsymbol{a}, \boldsymbol{\psi} \mid \boldsymbol{r})$ can be factored as

$$
\begin{aligned}
& p(\boldsymbol{a}, \boldsymbol{\psi} \mid \boldsymbol{r}) \propto\left[\prod_{k=1}^{K} P\left(\psi_{0}^{(k)}\right)\right] \prod_{n=0}^{N-1} F_{n}^{\prime}\left(\boldsymbol{\psi}_{n}\right) \\
& \prod_{k=1}^{K} U_{n}^{(k)}\left(\psi_{n}^{(k)}\right) G_{n}^{(k)}\left(\psi_{n+1}^{(k)}, \psi_{n}^{(k)}, a_{n}^{(k)}\right) P\left(a_{n}^{(k)}\right) .
\end{aligned}
$$

where

$$
\begin{aligned}
& F_{n}^{\prime}\left(\psi_{n}\right)=\prod_{k=1}^{K-1} \prod_{i=k+1}^{K} \exp \left\{-\frac{1}{\xi^{2}} \Re\left(e^{j\left(\psi_{n}^{(k)}-\psi_{n}^{(i)}\right)}\right)\right\} \\
& U_{n}^{(k)}\left(\psi_{n}^{(k)}\right)=\exp \left\{\frac{1}{\xi^{2}} \Re\left(x_{n}^{(k)} e^{-j \phi_{n}^{(k)}}\right)\right\} \\
& G_{n}^{(k)}\left(\psi_{n+1}^{(k)}, \psi_{n}^{(k)}, a_{n}^{(k)}\right)=v\left(\psi_{n+1}^{(k)}-\psi_{n}^{(k)}-\pi h a_{n}^{(k)}\right) .
\end{aligned}
$$

The stochastic model for $h_{e}^{(k)}$ depends on the considered standard. As an example, in the case of Bluetooth BR [4] $h_{e}^{(k)}$ follows a uniform distribution in the interval $[0.28-h, 0.35-$ $h]$, whereas in the case of the AIS standard [6], it follows a Gaussian distribution with mean $0.5-h$ and variance $\sigma_{h_{e}}^{2}$. Assuming that $h_{e}^{(k)}$ has mean zero (this is always possible by properly choosing $h$ ) and an even probability density function (pdf), given $a_{n}^{(k)}$ the random variable $\pi h_{e}^{(k)} a_{n}^{(k)}$ is statistically equivalent to $\pi h_{e}^{(k)}$. We will also assume that the random variables $y_{n}^{(k)}=\Delta_{n}^{(k)}+\pi h_{e}^{(k)}$ are independent, ${ }^{3}$ although this is clearly not true. The pdf $v\left(y_{n}^{(k)}\right)$ of the random variable $y_{n}^{(k)}$ can be easily computed from the a-priori information available at the receiver (i.e., using the distributions of $\Delta_{n}^{(k)}$ and $h_{e}^{(k)}$ and the fact that they are independent). As an example, when $h_{e}^{(k)}$ has uniform distribution in $[-\epsilon, \epsilon]$, we have

$$
v\left(y_{n}\right)=\frac{1}{2 \pi \epsilon}\left[\mathrm{Q}\left(\frac{y_{n}-\pi \epsilon}{\sigma_{\Delta}}\right)-\mathrm{Q}\left(\frac{y_{n}+\pi \epsilon}{\sigma_{\Delta}}\right)\right]
$$

where $\mathrm{Q}(x)=\frac{1}{\sqrt{2 \pi}} \int_{x}^{+\infty} e^{-\frac{u^{2}}{2}} d u$. The FG corresponding to (14) has cycles of length four. As shown in the previous section we can remove these short cycles by stretching the variables $a_{n}^{(k)}$ and $\psi_{n}^{(k)}$ in $\left(a_{n}^{(k)}, \psi_{n}^{(k)}\right)$ and thus obtaining a graph with shortest cycles of length twelve. As in [2], we assume that the interference among non-adjacent users is negligible. Then, $F_{n}^{\prime}\left(\boldsymbol{\psi}_{n}\right)$ in (15) can be approximated as

$$
F_{n}^{\prime}\left(\boldsymbol{\psi}_{n}\right) \simeq \prod_{k=1}^{K-1} F_{n}^{\prime(k, k+1)}\left(\psi_{n}^{(k)}, \psi_{n}^{(k+1)}\right)
$$

${ }^{3}$ This assumption corresponds to the case when $h_{e}^{(k)}$ can assume independent values in different symbol intervals.

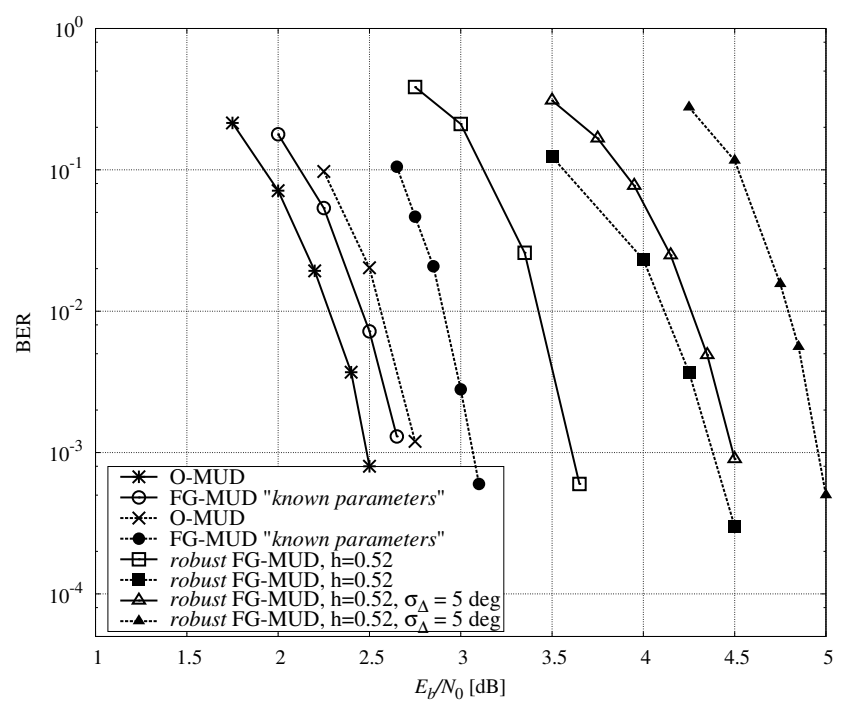

Fig. 2. Serial concatenation with CC $(5,7)$ via a pseudo-random interleaver with length 2048 bits with MSK modulation and a channel spacing $F=0.5$ for two different number of users $K=3$ (solid curves) and $K=5$ (dashed curves)

where

$F_{n}^{\prime}(k, k+1)\left(\psi_{n}^{(k)}, \psi_{n}^{(k+1)}\right)=\exp \left\{-\frac{1}{\xi^{2}} \Re\left(e^{j\left(\psi_{n}^{(k+1)}-\psi_{n}^{(k)}\right)}\right)\right\}$.

The resulting FG is shown in Figure 1.

\section{Simulation Results}

We compare three detectors. The first one, referred to as O-MUD, is the optimal multi-user detector. The second one, referred to as FG-MUD for factor graph multi-user detection, was proposed in [2]. The third one is the proposed robust FG-MUD. We consider the serial concatenation, through a pseudo-random interleaver of length 2048 bits, with a binary convolutional encoder with generators $(7,5)$ (octal notation). The bit error rate performance is averaged over all users. An oversampling factor $N_{s}=10$ is considered. We assume that all users transmit at the same power and share the same modulation format and that channels are equally spaced in frequency. For all considered receivers, a number of 12 iterations between detector and decoder is allowed. The first example corresponds to a minimum shift keying (MSK) modulation format (binary modulation with $h=1 / 2$ and a rectangular frequency pulse of duration $T$ ). The normalized spacing $F=\left|f^{(k+1)}-f^{(k)}\right|$ is equal to 0.5 . Two different numbers of users are considered $K=\{3,5\}$. The O-MUD and FG-MUD performance are plotted as reference $\left(h^{(k)}=1 / 2\right)$. Concerning the "robust FGMUD”, we assume $h^{(k)}=0.52$ for all users and the detection considers that the modulation index of each user takes on value randomly and uniformly in the interval $[0.45,0.55]$. From Fig. 2 , in the presence of modulation index mismatch only, at BER $=10^{-3}$ the "robust FG-MUD" performs at around 1 $\mathrm{dB}$ (resp $1.4 \mathrm{~dB}$ ), compared to the FG-MUD, for $K=3$ (resp $K=5$ ). In the presence of a phase noise with standard deviation $\sigma_{\Delta}=5 \mathrm{deg}$ in addition to the modulation index 


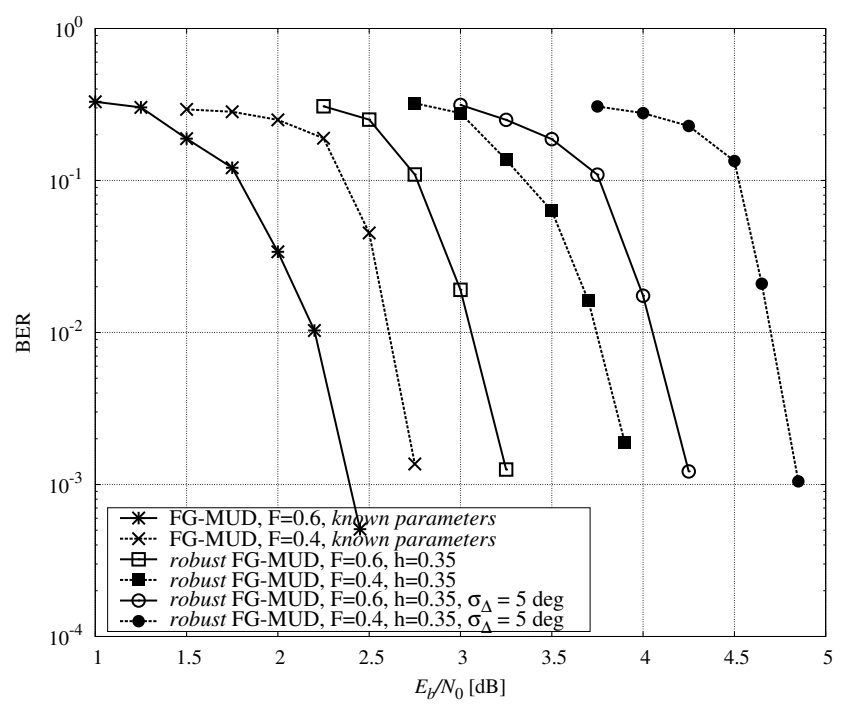

Fig. 3. Serial concatenation with CC $(5,7)$ via a pseudo-random interleaver with length 2048 bits with 2 GFSK modulation with $\beta=0.5$ and $h=1 / 3$, for a number of users $\mathrm{K}=3$ and two different channel spacing $F=0.6$ (solid curves) and $F=0.4$ (dashed curves)

mismatch, we observe a degradation of $1.35 \mathrm{~dB}$ (resp 1.85 $\mathrm{dB}$ ), compared to the FG-MUD, for $K=3$ (resp $K=5$ ) at $\mathrm{BER}=10^{-3}$. In the second example, the same parameters for Bluetooth (BR) system as in [14] are taken, namely Gaussian frequency-shift keying (GFSK) modulation with $\beta=0.5$ and a frequency pulse duration $L=2$, whereas the modulation index $h$ can vary between 0.28 and 0.35 . We fix the number of users to $K=3$ and we consider both cases: $F=0.4$ and $F=0.6$. The FG-MUD performance are plotted with the nominal modulation index $\left(h^{(k)}=1 / 3\right)$. For the "robust FG-MUD", we assume $h^{(k)}=0.35$ and that the modulation index of each takes on value randomly and uniformly in the interval $[0.28,0.35]$. From Fig 3 , in the presence of modulation index mismatch only the proposed algorithm performs at 0.7 $\mathrm{dB}($ resp $1.15 \mathrm{~dB})$ compared to the FG-MUD, for $F=0.6$ (resp $F=0.4$ ) at $\mathrm{BER}=10^{-3}$. In the presence of a phase noise with standard deviation $\sigma_{\Delta}=5 \mathrm{deg}$ in addition to the modulation index mismatch, we observe a degradation of 1.9 $\mathrm{dB}($ resp $2.5 \mathrm{~dB})$, compared to the FG-MUD, for $F=0.6$ (resp $F=0.4)$ at $\mathrm{BER}=10^{-3}$.

\section{CONCLUSION}

In this paper, we have derived a robust multiuser binary CPM detector for FDM-CPM systems where the spectral efficiency (SE) of frequency-division-multiplexed (FDM) systems can be increased by reducing the spacing between adjacent users. Each user's binary CPM signal is generated through a low-cost transmitter, which leads to a modulation index error, and transmitted over a channel affected by phase noise. The proposed algorithm has been derived based on the Laurent representation of a CPM signal where only the principal component has been considered. Detection in the presence of an unknown modulation index and a time-varying phase noise is performed based on the sum-product algorithm working on a properly defined factor graph (FG). Performance in terms of BER are compared to the FG-MUD detector where it's shown that an acceptable performance is obtained even in the presence of a significant phase noise and modulation index uncertainty.

\section{REFERENCES}

[1] A. Barbieri, D. Fertonani, and G. Colavolpe, "Spectrally-efficient continuous phase modulations," IEEE Trans. Wireless Commun., vol. 8, pp. 1564-1572, Mar. 2009.

[2] A. Piemontese and G. Colavolpe, "A novel graph-based suboptimal multiuser detector for FDM-CPM transmissions," IEEE Trans. Wireless Commun., vol. 9, pp. 2812-2819, Sep. 2010.

[3] F. R. Kschischang, B. J. Frey, and H.-A. Loeliger, "Factor graphs and the sum-product algorithm," IEEE Trans. Inform. Theory, vol. 47, pp. 498-519, Feb. 2001.

[4] "Specification of the Bluetooth System," Dec. 2010, Bluetooth Special Interest Group document.

[5] ETSI, "Digital Enhanced Cordless Telecommunication (DECT); Ultra low Energy (ULE); Machine to Machine Communications; Part 1: Home Automation Network (phase 1)," 2013

[6] D. Bonacci, J.-P. Millerioux, R. Prevost, J. Lemaitre, M. Coulon, and J.Y. Tourneret, "Advanced concepts for satellite reception of AIS messages (Toulouse Space Show, Toulouse, 25/06/2012-28/06/2012)," 2012.

[7] P. A. Laurent, "Exact and approximate construction of digital phase modulations by superposition of amplitude modulated pulses (AMP)," IEEE Trans. Commun., vol. 34, pp. 150-160, Feb. 1986.

[8] G. Colavolpe and R. Raheli, "Reduced-complexity detection and phase synchronization of CPM signals," IEEE Trans. Commun., vol. 45, pp. 1070-1079, Sep. 1997

[9] A. Barbieri and G. Colavolpe, "Simplified soft-output detection of CPM signals over coherent and phase noise channels," IEEE Trans. Wireless Commun., vol. 6, no. 7, pp. 2486-2496, Jul. 2007.

[10] H. Meyr, M. Oerder, and A. Polydoros, "On sampling rate, analog prefiltering, and sufficient statistics for digital receivers," IEEE Trans. Commun., vol. 42, pp. 3208-3214, Dec. 1994.

[11] G. Colavolpe and G. Germi, "On the application of factor graphs and the sum-product algorithm to ISI channels," IEEE Trans. Commun., vol. 53, pp. 818-825, May 2005.

[12] M. Messai, G. Colavolpe, K. Amis, and F. Guilloud, "Robust Detection of Binary CPMs with Unknown Modulation Index," IEEE Commun. Letters, vol. 19, no. 3, pp. 339-342, Mar. 2015.

[13] D. Xu and Y. Zhang, "Estimation of the modulation index of CPM signals based on Laurent's decomposition," IEEE Trans. Wireless Commun., vol. 12 , no. 12, pp. 6268-6280, Dec. 2013.

[14] L. Lampe, R. Schober, and M. Jain, "Noncoherent sequence detection receiver for Bluetooth systems," IEEE J. Select. Areas Commun., vol. 23, no. 9, Sep. 2005. 